\title{
Shadowgrams of a Dense Micro-Capillary Plasma Obtained with a Table-Top Soft X-Ray Laser
}

\author{
C. H. Moreno, M. C. Marconi, V. N. Shlyaptsev, and J. J. Rocca, Senior Member, IEEE
}

\begin{abstract}
A sequence of high resolution shadowgrams that map the evolution of the plasma of a $380 \mu \mathrm{m}$ diameter microcapillary discharge was obtained using a very compact $46.9 \mathrm{~nm}$ laser. These images are the first plasma diagnostics data obtained using a table-top soft X-ray laser.
\end{abstract}

Index Terms - Capillary discharges, shadowgraphy, soft X-ray diagnostics, soft X-ray lasers.

D UE to their short wavelength, high brightness, short pulse duration, and high degree of collimation, soft X-ray lasers are excellent radiation sources to perform shadowgraphy studies in dense plasmas. Recently, a Ne-like Y soft Xray laser pumped by the Nova laser was used to image with micrometer-scale resolution laser-accelerated and laserexploded foils excited by a separate beam of the same laser facility [1]. Now the advent of table-top soft X-ray lasers with output pulse energy up to $25 \mu \mathrm{J}$ [2], [3] has opened the possibility of utilizing soft X-ray laser beams to probe a wide variety of dense plasmas. We have obtained a sequence of high-resolution shadowgrams that map the evolution of the plasma of a micro-capillary discharge using a table-top soft $\mathrm{X}$-ray laser as backlighter. Micro-capillary plasmas created by discharge [4], [5] or laser [6] excitation are of interest as plasma waveguides. The two-dimensional (2-D) soft X-ray shadowgrams have a spatial resolution of about $5 \mu \mathrm{m}$ and a time resolution of $0.6-0.7 \mathrm{~ns}$.

The micro-capillary plasma that was the subject of this study has been generated by exciting a $380 \mu \mathrm{m}$ diameter, $8 \mathrm{~mm}$ long evacuated channel in polyacetal with a relatively fast current pulse. The plasma was created from material ablated from the capillary walls by the current pulse, which had an amplitude of about $1.5 \mathrm{kA}$ and a half period of $155 \mathrm{~ns}$. The micro-capillary discharge plasma channel was illuminated on-axis by a low divergence beam of $46.9 \mathrm{~nm}$ wavelength radiation produced by a discharge-pumped Ne-like Ar tabletop laser. This laser emits pulses of $0.6-0.7 \mathrm{~ns}$ full width at half maximum duration in a single strong laser line at $46.9 \mathrm{~nm}$ [3]. The imaging setup employed to record the shadowgrams is schematically illustrated in the top of Fig. 1. The microcapillary discharge was placed at $60 \mathrm{~cm}$ from the exit aperture

Manuscript received July 1, 1998; revised September 18, 1998. This work was supported by the Department of Energy Grant DE-FG03-98DP00208 and the National Science Foundation.

C. H. Moreno and M. C. Marconi are with the Department of Electrical Engineering, Colorado State University, Fort Collins, CO 80523 USA, on leave from Universidad de Buenos Aires, Depto de Fisica, Argentina (e-mail: rocca@engr.colostate.edu).

V. N. Shlyaptsev and J. J. Rocca are with the Department of Electrical Engineering, Colorado State University, Fort Collins, CO 80523 USA.

Publisher Item Identifier S 0093-3813(99)02410-8. of the soft X-ray laser. The plasma was imaged onto a 2-D soft X-ray sensitive detector consisting of a micro channelplate and phosphor screen using a $60 \mathrm{~cm}$ radius of curvature concave iridium-coated mirror. A plane iridium-coated mirror was used to relay the image. The magnification of the imaging/detector system was $\approx 24 \mathrm{x}$. The spatial resolution of the entire imaging system was measured to be $\approx 5 \mu \mathrm{m}$ at the object plane by imaging an array of $25 \mu \mathrm{m}$ diameter pinholes placed at the exit plane of the capillary channel.

Fig. 1(b) shows a sequence of soft X-ray shadowgrams describing the temporal evolution of the micro-capillary plasma. The first image $(0 \mathrm{~ns})$ of the sequence was obtained by propagating the soft-X-ray laser pulse through the capillary before the initiation of the discharge current pulse, and therefore corresponds to the situation in which no plasma is present. A relatively uniform illumination of the capillary channel is observed. The second (26 ns) and third (39 ns) images illustrate the initial phase of the plasma evolution, corresponding to times shortly after the discharge breakdown. Dense regions of ablated material that strongly absorb the soft X-ray laser radiation are observed to develop at a few locations on the wall perimeter. This indicates that during the initial phase of the discharge a significant fraction of the current flow is predominantly localized in a few surface discharge channels that rapidly expand. However, as the current increases and the plasma expands toward the evacuated center of the capillary the nonuniformities decrease. After $\approx 80 \mathrm{~ns}$ from the initiation of the current pulse the boundary between the absorbent and transparent plasma regions is observed to become highly symmetric. This boundary is measured to move toward the capillary axis with a velocity of $5 \times 10^{4} \mathrm{~cm} / \mathrm{s}$ and $1 \times$ $10^{5} \mathrm{~cm} / \mathrm{s}$ at 50 and $100 \mathrm{~ns}$ after the onset of the current pulse, respectively. The spectra of the micro-capillary plasma in the 50-120 $\mathrm{nm}$ region shows emission from carbon ions with degree of ionization ranging from $\mathrm{C} I \mathrm{I}$ to $\mathrm{C}$ IV and from oxygen atoms ionized up to O VI. The observation of emission from $\mathrm{C}$ II ions, that have an ionization potential lower than the $26.5 \mathrm{eV}$ energy of the laser photons, suggest in agreement with our model calculations that the major part of the laser attenuation in the cold outer annular plasma region is due to photoionization of weakly ionized atoms. In the central region the plasma is less dense, hotter with a temperature of $\approx 15 \mathrm{eV}$, and more highly ionized. The boundary between the absorbing and transparent regions is therefore mainly determined by the distribution of the low charge species. Our calculations show that the electron density, that has a concave profile with a minimum on axis, increases with time to exceed $2 \times 10^{19} \mathrm{~cm}^{-3}$ at the center of the capillary. 
(a)
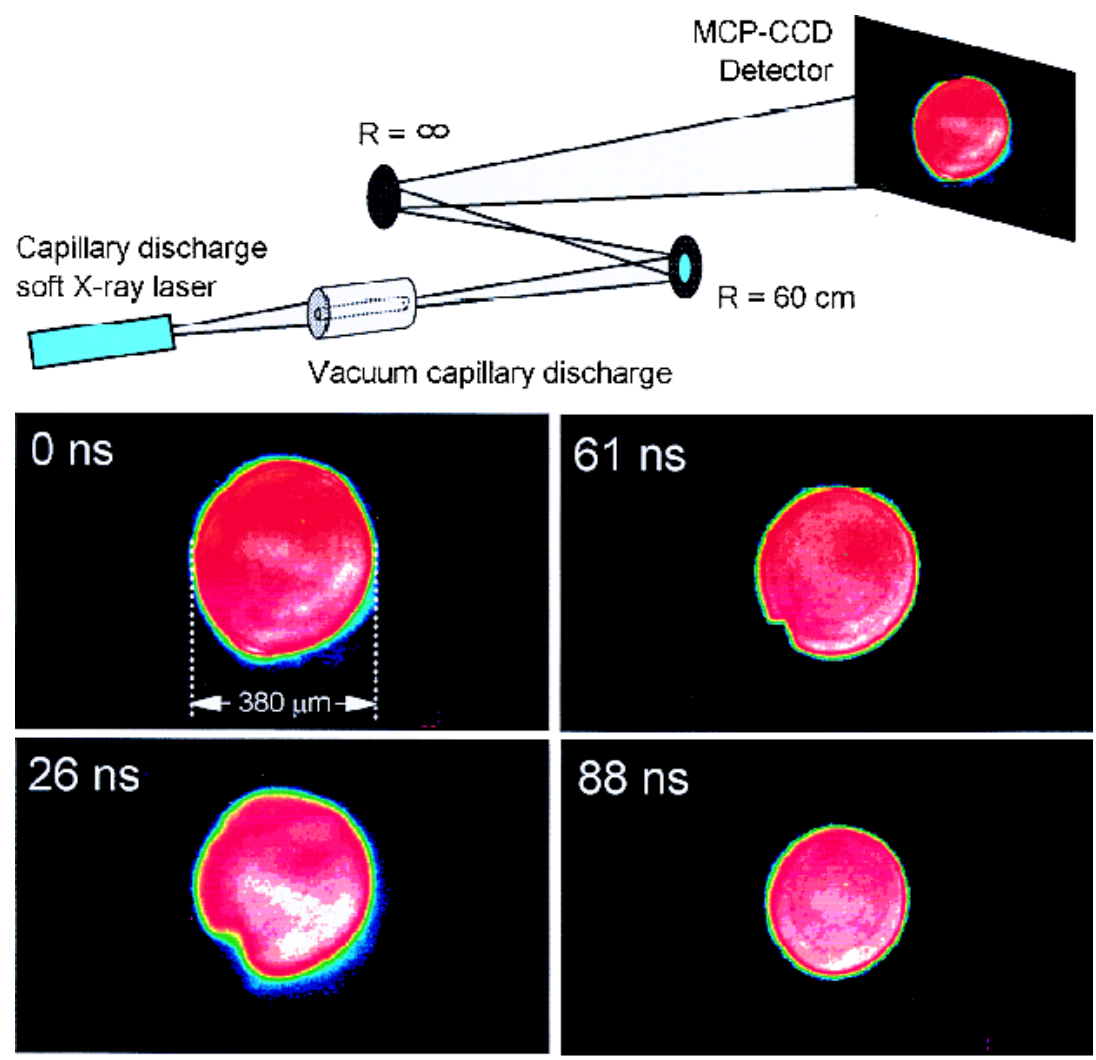

(b)
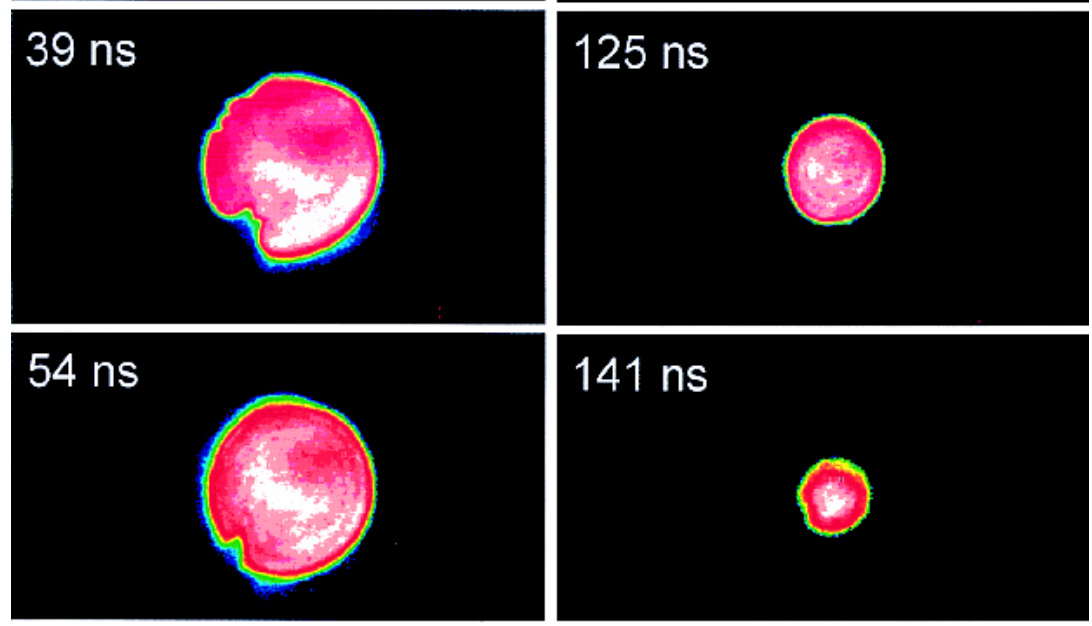

Fig. 1. (a) Schematic representation of the experimental setup. (b) Sequence of on-axis shadowgrams describing the evolution of the micro-capillary plasma The time of each shadowgram respect to the initiation of the current pulse is indicated. Lighter regions indicate lower absorption of the soft X-ray laser. In the darker regions the laser is attenuated by photo ionization of weakly ionized ions and in a lesser extent by free-free absorption.

In summary, we have used a very compact dischargepumped $46.9 \mathrm{~nm}$ laser to perform soft X-ray laser shadowgraphy of a dense plasma created by a vacuum discharge in a micro-capillary channel, obtaining new insight in the intriguing physics of these plasmas. This is to our knowledge the first demonstration of plasma diagnostics with a table-top soft Xray laser.

\section{REFERENCES}

[1] R. Cauble, L. B. Da Silva, T. W. Barbee Jr., P. Celliers, J. C. Moreno, and A. S. Wan, "Micron-resolution radiography of laser-accelerated and X-ray heated foils with an X-ray laser," Phys. Rev. Lett., vol. 74, p. $3816,1995$.
[2] J. J. Rocca, D. P. Clark, J. L. A. Chilla, and V. N. Shlyaptsev, "Energy extraction and achievement of the saturation limit in a discharge-pumped table-top soft X-ray amplifier," Phys. Rev. Lett., vol. 77, p. 1476, 1996.

[3] B. R. Benware, C. H. Moreno, D. J. Burd, and J. J. Rocca, "Operation and output pulse characteristics of an extremely compact capillarydischarge tabletop soft X-ray laser," Opt. Lett., vol. 22, p. 796, 1997.

[4] Y. Ehrlich, C. Cohen, A. Zigler, J. Krall, P. Sprangle, and E. Esarey, "Guiding of high intensity laser pulses in straight and curved plasma channel experiments," Phys. Rev. Lett., vol. 77, p. 4186, 1996.

[5] J. J. Rocca, F. Tomasel, M. C. Marconi, J. L. A. Chilla, C. H. Moreno, B. R. Benware, V. Shlyaptsev, and J. J. Gonzalez, "Lasing in Ne-like S and other new developments in capillary discharge ultrashort wavelength lasers," SPIE J., vol. 3156, p. 164, 1997.

[6] D. V. Korobkin, C. H. Nam, S. Suckewer, and A. Goltsov, "Demonstration of soft X-ray lasing to ground state in Li III," Phys. Rev. Lett, vol. 77, p. 5206, 1996. 